\title{
Remote sensing of low-energy SEPs via charge exchange
}

\author{
H. S. Hudson*,†, A. L. MacKinnon ${ }^{\dagger}$ and N. R. Badnell** \\ ${ }^{*}$ Space Sciences Laboratory, UC Berkeley \\ 7 Gauss Way, Berkeley CA 94720, USA \\ †niversity of Glasgow \\ ${ }^{* *}$ University of Strathclyde
}

\begin{abstract}
Charge-exchange reactions at high energies provide new channels for the remote sensing of solar high-energy particles, as demonstrated by the recent detection of 1.8-5 MeV hydrogen atoms from a solar flare [1]. Orrall and Zirker [2] had earlier proposed the detection of low-energy protons via charge-exchange atomic reactions in the solar atmosphere, leading in the simplest case to extended red-wing emission in the Lyman-alpha line. We discuss the analogous process for the He II $304 \AA$ line (for alpha particles) and also assess the feasibility of the analogous process in the solar wind, whereby ambient $\mathrm{He}$ and $(\mathrm{C}, \mathrm{N}, \mathrm{O})$ ions allow low-energy alpha particles to undergo resonant charge exchange in the ambient corona and thereby produce $304 \AA$ wing emission close to the acceleration region.
\end{abstract}

Keywords: Flares, CMEs, Solar Energetic Particles

PACS: 96.60.qe, 96.60.ph, 96.60.Vg

\section{INTRODUCTION}

Solar energetic particles (SEPs) often appear near $1 \mathrm{AU}$ following eruptive flares and coronal mass ejections, and they may have significant consequences for human activities in space. The energetic electrons have strong electromagnetic signatures, and thus can be remotely sensed by radio or X-ray observations. The proton or heavy ion components of an SEP have no such direct coupling. On the other hand their charge-exchange reactions with other neutral particles or ions can in principle lead to similarly direct signatures. This provided the original means for the identification of the proton aurora, for example, in Vegard's classic 1939 work [3].

If we could detect SEP particles by remote-sensing techniques, we might learn a great deal about their acceleration physics. Kahler et al. [4] originally suggested the use of charge-exchange reactions for this purpose, and Kahler \& Ragot [5] later made additional suggestions regarding the interaction of SEPs with the solar wind, and possible signatures deriving therefrom, finding it possible to "rule out" SEP $\gamma$-ray signatures from the solar wind itself.

The present paper assesses the new systematic UV spectroscopy from the EUV Variability Experiment (EVE) on the Solar Dynamics Observatory (SDO) spacecraft [6] in this context. Charge exchange can happen in the corona or solar wind, as particles accelerated in a CME-driven shock wave escape into the heliosphere, via interactions with ambient ions, mainly $\mathrm{He}, \mathrm{C}, \mathrm{N}$, and $\mathrm{O}$ at alpha-particle energies of $0.5-5 \mathrm{MeV}$. These interactions lead to prompt radiative recombination and the formation of broad line wings. We note that these recombina- tion photons can escape from the lower corona, or penetrate it from sources behind the Sun, at heights where ENAs would be lost due to collisional stripping. We can estimate a "stripping height" from $\xi \sigma_{i} \approx 1$, where $\sigma_{i}$ is cross-section for collisional ionization and $\xi$ is the column density above this height. For $1 \mathrm{MeV}$ we find this to be 1.5-3 $\mathrm{R}_{\odot}$, using the Bargouty [7] cross-section in the Withbroe [8] density models.

\section{ALPHA PARTICLES IN THE CHROMOSPHERE}

In standard flare theory, particle acceleration in the corona leads to bombardment of the lower solar atmosphere by energetic particles, producing effects seen in "footpoint" sources [e.g., 9]. Generally the flare $\gamma$-ray emission shows the ion bombardment, and the hard Xray bremsstrahlung the electron bombardment. The energy losses by the stopping particles heat and ionize the medium, but at least initially the highest-energy particles can enter neutral or weakly ionized regions of the chromosphere and photosphere. Because the particle bombardment is intense, the ionization will increase and quench the charge-exchange reactions, but for a time they can proceed and create the mainly red-wing emission. In the simplest geometry (vertical incidence and viewing), protons in the energy range $0.1-1 \mathrm{MeV}$ will produce redshifts of $18-56 \AA$ in the Ly- $\alpha$ line of hydrogen. Even with allowances for pitch-angle distributions and oblique viewing angles, it is clear that even low-energy primaries map onto large Doppler shifts of the recombining ENAs. Accordingly this process has great significance: not only 
could one remotely detect the high-energy particles, but one could detect them even at energies below the thresholds for $\gamma$-ray production. Unfortunately, suitable solar observations have not been available, and the best reported non-solar case [10] was only marginally significant.

The analogous process for primary alpha particles and the $\mathrm{He}$ II Ly- $\alpha$ line at $304 \AA$ was discussed in detail by Peter et al. [11], and for X-rays by Raymond [12]. The range of particle energies from 0.1-1.0 MeV/nucleon maps to shifts $\Delta \lambda \approx 4-14 \AA$ relative to $304 \AA$. The EVE MEGS-A spectrometer [6] has a spectral resolution of about $1 \AA$, with excellent coverage, time resolution, and photometric precision via 10-s sampling of integrated sunlight. Unfortunately these data do not show evidence for the expected red-wing enhancement, even in six X-class flares [13]. The many assumptions required in the theoretical estimates make it difficult to interpret the EVE upper limits in terms of particles in a modelindependent manner, but the upper limits are severe. We have no reason to doubt the existence of alpha particles in many of these large flares, particularly those from which $\gamma$-ray lines were detected, so this is a distinct puzzle.

\section{ALPHA PARTICLES IN THE SOLAR WIND - THEORY}

The charge-exchange reactions at high energies in the solar wind may pose simpler theoretical problems, e.g. in that the ionization states in the ambient medium may not be affected by the SEPs themselves. Given $N$

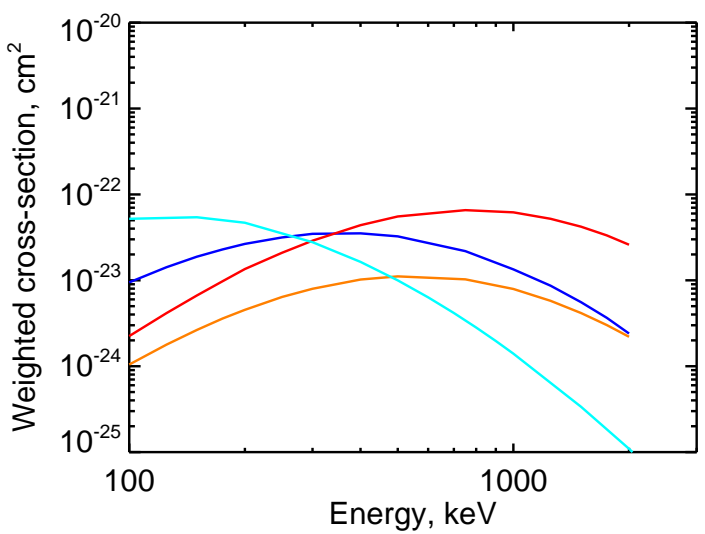

FIGURE 1. Net cross-sections weighted to hydrogen: He, pale blue; $\mathrm{O}$, red; $\mathrm{C}$, blue; $\mathrm{N}$, gold. The weighting includes coronal abundances for these elements, their ionization fractions for a $1.5 \mathrm{MK}$ equilibrium, and an estimated factor 0.2 to account for recombination to an excited state leading to Ly $\alpha$ emission. particles accelerated close to the Sun, we crudely estimate the fluence of recombination photons as $F=$ $N \xi \sigma /\left(4 \pi A U^{2}\right)$ photons $\mathrm{cm}^{-2}$, for a corona/solar wind column density $\xi$ particles $\mathrm{cm}^{-2}$ [e.g., 8]; here $\sigma$ represents the weighted cross-section as illustrated in Figure 1. We have used cross-sections for He-like ions from Kuang [14], with standard coronal elemental abundances and the Mazzotta et al. [15] ionization-equilibrium calculation for a representative temperature of $1.5 \mathrm{MK}$. At $3 \mathrm{R}_{\odot}, \xi \approx 10^{16}$ particles $\mathrm{cm}^{-2}$; from the Figure, the weighted cross-section $\sigma \approx 10^{-22} \mathrm{~cm}^{2}$. Hence for $\mathrm{N}=$ $10^{35}$ alpha particles, we would estimate a $1-2 \mathrm{MeV}$ fluence of about 200 photons $\mathrm{cm}^{-2}$, which falls 7-8 decades below EVE's sensitivity, as described below.

\section{ALPHA PARTICLES IN THE SOLAR WIND - OBSERVATIONS}

In spite of this pessimistic conclusion regarding the detectability of charge-exchange line wings, we have carried out a search in the EVE data. At the time of writing, the strongest SEP event was that associated with the flare SOL2012-01-23T03:59 (M8.7), which also had an extremely rapid initial rise. The X-ray event was not observed by RHESSI; Fermi missed the flare onset, but did observe a "soft-hard-hard" late phase of the type correlated with SEP production $[16,17]$ following the GOES maximum.

The charge-exchange emission should appear in the vicinity of the line, in the form of broadened wings whose shape depends upon the velocity distribution function of the ions, as described above. Figure 2 shows a search for such an effect, with time series for bluewing spectral positions corresponding to $0.1,1.0$, and $0.3 \mathrm{MeV} /$ nucleon (from the bottom of the Figure). The Figure indeed shows spectral enhancements outside the core of the line, but these are not distributed with $\Delta \lambda$ as expected for the charge-exchange signature. The spectral distribution and time dependence of the broad wings thus seem improbable for the shock-acceleration picture, and the implied fluxes greatly exceed our estimates. The variations that do appear probably reflect flare-associated lines and continua, sources of background that would be greatly diminished via imaging and by higher spectral resolution than EVE can supply.

\section{CONCLUSIONS}

The EVE spectroscopy has given us a first chance to search for the charge-exchange excitation of the wings of the He II Ly- $\alpha$ line at $304 \AA$. This had been suggested by Orrall \& Zirker [2] as resulting from the chromospheric 


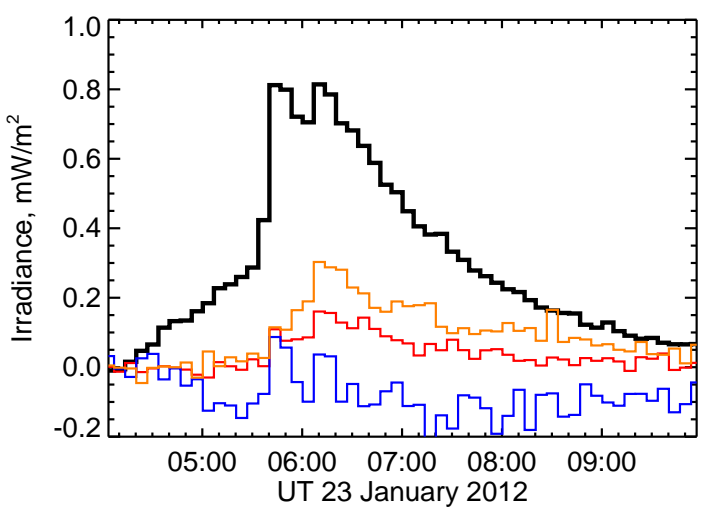

FIGURE 2. The $304 \AA$ line time series during the SEP flare SOL2012-01-23: line center and blue-wing points over at line-of-sight velocities corresponding to $0.1,0.3$, and 1.0 MeV/nucleon. The background level is derived from the time interval 03:00:11-03:03:21 UT and the line-wing excess irradiances increased by $100 \times$ for clarity.

interactions during thick-target precipitation, but our previous paper [13] showed no signs of this effect. In this paper we further suggest that the charge-exchange line wings could be detectable in the solar corona (or solar wind) via the interactions of the alpha particles with ambient ions, mainly $\mathrm{He}, \mathrm{C}, \mathrm{N}$, and $\mathrm{O}$, for which the chargeexchange reaction can be resonant. We have indeed also failed to detect such a coronal source in the eruptive event SOL2012-01-23, the best example of an SEP event in the current EVE database; we find that EVE's sensitivity is much too low for this application. A sensitive imaging spectrograph would have a good chance at detecting SEPs at their point of acceleration, however; the EUV fluxes resulting from charge exchange do not suffer from stripping, as do the ENAs, and so in principle the photons can probe deeper into the corona. This would give a major advantage if the acceleration happened below the stripping height, some $1.5-3 \mathrm{R} \odot$ radial distance.

The search for charge-exchange signatures of highenergy particles in EVE and other spectroscopic databases should continue, if only because of the suggestion of broad wings of the He II $304 \AA$ line in Figure 2. Furthermore, as [4] put it: "we currently have little information about where and when shock acceleration of SEPs occurs in the corona and interplanetary medium" - still true! A dedicated search by a sensitive EUV instrument may eventually be able to solve this problem.

\section{ACKNOWLEDGMENTS}

Author H. Hudson thanks NASA for support under contract NAS 5-98033, author MacKinnon for STFC rolling grant ST/I001808/1, and author Badnell for APAP Network grant ST/J000892/1.

\section{REFERENCES}

1. R. A. Mewaldt, R. A. Leske, E. C. Stone, A. F. Barghouty, A. W. Labrador, C. M. S. Cohen, A. C. Cummings, A. J. Davis, T. T. von Rosenvinge, and M. E. Wiedenbeck, Astrophysical Journal (Letters) 693, L11-L15 (2009).

2. F. Q. Orrall, and J. B. Zirker, Astrophysical Journal 208, 618-632 (1976).

3. L. Vegard, Nature 144, 1089-1090 (1939).

4. S. W. Kahler, J. C. Raymond, and J. M. Laming, "Spectroscopic diagnostics for remote detection of particle acceleration regions at coronal shocks," in American Institute of Physics Conference Series, edited by S. R. Habbal, R. Esser, J. V. Hollweg, \& P. A. Isenberg, 1999, vol. 471 of American Institute of Physics Conference Series, pp. 685-688.

5. S. W. Kahler, and B. R. Ragot, Astrophysical Journal 675, 846-852 (2008).

6. T. N. Woods, F. G. Eparvier, R. Hock, A. R. Jones, D. Woodraska, D. Judge, L. Didkovsky, J. Lean, J. Mariska, H. Warren, D. McMullin, P. Chamberlin, G. Berthiaume, S. Bailey, T. Fuller-Rowell, J. Sojka, W. K. Tobiska, and R. Viereck, Solar Physics 275, 115-143 (2012).

7. A. F. Barghouty, Physical Review A 61, 052702 (2000).

8. G. L. Withbroe, Astrophysical Journal 325, 442-467 (1988).

9. L. Fletcher, B. R. Dennis, H. S. Hudson, S. Krucker, K. Phillips, A. Veronig, M. Battaglia, L. Bone, A. Caspi, Q. Chen, P. Gallagher, P. T. Grigis, H. Ji, W. Liu, R. O. Milligan, and M. Temmer, Space Science Reviews 159, 19-106 (2011), 1109.5932.

10. B. E. Woodgate, J. C. Brandt, M. W. Kalet, P. J. Kenny, E. A. Tandberg-Hanssen, E. C. Bruner, J. M. Beckers, W. Henze, E. D. Knox, and C. L. Hyder, Solar Physics 65, 73-90 (1980).

11. T. Peter, E. N. Ragozin, A. M. Urnov, D. B. Uskov, and D. M. Rust, Astrophysical Journal 351, 317-333 (1990).

12. J. C. Raymond, Astronomische Nachrichten 333, 305 (2012).

13. H. S. Hudson, L. Fletcher, A. L. MacKinnon, and T. N. Woods, Astrophysical Journal 752, 84 (2012).

14. Y. R. Kuang, Journal of Physics B: Atomic, Molecular and Optical Physics 25, 199 (1992), URL http: / / stacks.iop.org/0953-4075/25/i=1/a=023.

15. P. Mazzotta, G. Mazzitelli, S. Colafrancesco, and N. Vittorio, Astronomy \& Astrophysics Supplement 133, 403-409 (1998), arXiv:astro-ph/9806391.

16. A. L. Kiplinger, Astrophysical Journal 453, 973 (1995).

17. J. A. Grayson, S. Krucker, and R. P. Lin, Astrophysical Journal 707, 1588-1594 (2009). 